\title{
Comunidade de abelhas nativas (Apidae) em Floresta Ombrófila Densa Montana em Santa Catarina
}

\author{
Denise M.D.S. Mouga ${ }^{1} \&$ Cristiane $\mathrm{Krug}^{2}$
}

\author{
${ }^{1}$ Departamento de Ciências Biológicas, Universidade da Região de Joinville. Campus Universitário, Caixa Postal 246, \\ 89201-972 Joinville, Brasil. E-mail: dmouga@terra.com.br \\ 2 Programa de Pós-Graduação em Entomologia, Universidade de São Paulo. Avenida Bandeirantes 3900, Bloco 2, \\ 14040-901 Ribeirão Preto, Brasil. E-mail: criskrug@usp.br
}

\begin{abstract}
Community of native bees (Apidae) in a tropical rain forest mountain area in Santa Catarina. This study evaluated the bee community and their associated melliferous plants in the northern region of the state of Santa Catarina, Brazil in order to ascertain the diversity and floral interactions of the local Apidae. Twelve samplings were conducted (April 2006 to March 2007) in transects, where nets were used to capture bees directly from their flowers. Voucher specimens for both bees and plants were deposited at UNIVILLE. Diversity and equitability indexes were calculated. Our sampling effort resulted in a total of 934 bees in 72 genera and five families. Forty-four plant species belonging to 19 families were visited. A dominant family in Southern Brazil, Asteraceae, was visited most often (50\% of the taxa). Nearly $20 \%$ of the plants were visited by only one bee species. Meliponina, Ceratinina and Halictinae were prevalent. The diversity of the apifauna composition (Apinae followed by Halictinae) is typical for southern environments, with few abundant species and many singletons. The variation found for the equitability indexes revealed low intertaxa dominance, diversity oscillation and interspecies relative equilibrium. Values of richness and abundance indicate a transitional distribution pattern (subtropical to temperate). Rare taxa, the presence of new records for the state, and the collector's slope indicate an estimated greater richness.
\end{abstract}

KEY WORDS. Apifauna; conservation; community structure.

O conhecimento sobre a diversidade biológica existente atualmente em países mega-diversos é bastante extenso. Entretanto, apesar do esforço de pesquisa, este conhecimento ainda se apresenta fragmentado e com lacunas consideráveis. Muitos grupos taxonômicos de grande importância e diversidade são ainda pouco conhecidos, seja por coletas muito limitadas, seja por falta de trabalho taxonômico ou dificuldades em elucidar sua estrutura com métodos e ferramentas convencionais (LEWINSOHN 2001). Regiões geográficas e habitats foram investigados de forma muito desigual e sua representação em coleções biológicas espelha este fato. Encaixam-se nesta situação, as abelhas nativas e a região sul do Brasil, mais especificamente o estado de Santa Catarina.

As abelhas são importantes polinizadoras da flora nativa e desenvolvem relações interespecíficas importantes no grupo ao qual pertencem. Também mantêm interações com a flora melitófila. Todas essas interfaces revelam comunidades com estruturas de guildas. Estas inter-relações podem ser utilizadas como medida do status do ambiente e de sua conservação.

Nos meses seguintes à realização deste estudo, foi implantada a Pequena Central Hidroelétrica Rio Vermelho, na Área de Preservação Ambiental (APA) do Rio Vermelho, município de São Bento do Sul. A instalação de PCHs é fator de alteração ambiental considerável pelas consequências que acarreta (ToNHASCA JR 2005). Como a região em apreço conserva ainda ambientes bastante preservados (Medeiros 2002), foi empreendido o levantamento da comunidade de abelhas, tendo o propósito de conhecer a biodiversidade da apifauna, o compartilhamento de seus recursos e suas interelações florais, em uma região meridional de Mata Atlântica. Estes objetivos visam contribuir para o plano de manejo da APA, uma vez que as abelhas são consideradas relevantes bioindicadores, devido as relações que desenvolvem com a flora local (Kevan 1999).

No estado de Santa Catarina, levantamentos sobre a apifauna foram realizados, principalmente sob a forma de dissertações e teses, mas poucos foram publicados (OrTolan \& Laroca 1996, Steiner et al. 2006, Krug \& Alves-dos-Santos 2008).

Para a região Neotropical, temperada e subtropical, os táxons Halictinae, Apinae não corbiculados e Megachilinae apresentam a maior proporção de espécies, segundo Martins (1994). A análise dos inventários de abelhas realizados no sul do Brasil, mostram que a proximidade geográfica é importante para a união dos blocos de censos, o que sugere uma fauna regionalmente definida (C. Pinheiro-Machado com. pes.). Em 
geral, os trabalhos realizados nesta região do Brasil, mostram uma apifauna com maior diversidade de espécies de Apinae não corbiculados, seguido de Halictinae e Megachilinae (STEINER et al. 2006, PinHeiro-Machado et al. 2006, Alves-Dos-SANTOS 2007).

Nesse trabalho, foi realizado um levantamento das espécies de abelhas silvestres que ocorrem em Floresta Ombrófila Densa Montana, em uma região meridional de Mata Atlântica. Foram avaliados parâmetros de abundância relativa, riqueza, diversidade, equitabilidade, predominância de espécies e fenologia de forrageamento. As espécies da flora que são exploradas assim como a relação que se estabelece entre as plantas e sua melissofauna também foram analisadas.

\section{MATERIAL E MÉTODOS}

O estudo foi realizado no entorno do Centro de Estudos e Pesquisas Ambientais Rugendas, localizado na APA Rio Vermelho $\left(26^{\circ} 3^{\prime} \mathrm{S}, 49^{\circ} 3^{\prime} \mathrm{W}\right)$, município de São Bento do Sul, situado na região nordeste do estado de Santa Catarina. Essa região está inserida nos domínios de Mata Atlântica - Floresta Ombrófila Densa Montana (FODM) (altitude de $800 \mathrm{~m}$ ). Apresenta sazonalidade na distribuição das chuvas, com altos índices pluviométricos anuais (clima segundo Köeppen do tipo Cfa). O relevo constitui-se de morros e escarpas da Serra do Mar, com encostas íngremes e solo raso, algumas vezes rochoso (Medeiros 2002).

O método de obtenção dos dados baseou-se no trabalho de SAKagami et al. (1967). Assim, os espécimes de Apidae foram procurados visualmente dentro da área de estudo que se distribuía ao longo de um transecto previamente determinado de aproximadamente $3000 \mathrm{~m}$, percorrido durante períodos de seis horas, em cada dia de coleta, em percurso alternado a cada coleta. O transecto incluiu trilhas, clareiras, cortes de estrada e acessos diversos. O período de observação e coleta de espécimes se fez no horário entre 9:00 e 16:00 h (horário de maior insolação), em dias com condições meteorológicas favoráveis. As observações foram independentes do horário de verão implantado. Foram realizadas 12 amostragens diurnas, uma a cada mês, de abril de 2006 a março de 2007.

Os espécimes foram capturados por meio de dois tipos de redes entomológicas (malha de $1 \mathrm{~mm}$, diâmetro de $25 \mathrm{~cm}$ e malha de $0,1 \mathrm{~mm}$, diâmetro de $10 \mathrm{~cm}$ ), anestesiados e sacrificados com acetato de etila. Os exemplares foram preparados para identificação segundo Michener et al. (1994). Espécimes de Apis mellifera Linnaeus, 1758 que foram localizados forrageando, não foram coletados mas sua presença nas plantas foi anotada e registrada por estimativa quantitativa.

Os espécimes de Apoidea foram identificados em microscópio estereoscópico, com a ajuda de chaves dicotômicas da literatura especializada (Michener et al. 1994, Michener 2000, Silveira et al. 2002, Moure et al. 2007) e colaboração de especialistas. Adotou-se a classificação de Melo \& Gonçalves (2005).

As plantas sobre as quais foram capturados os espécimes de Apidae foram coletadas para identificação (ramos, flores e botões), afim de constituir exsicatas. Foram identificadas em microscópio estereoscópico, com a ajuda de literatura especializada e colaboração de especialistas.

Para cada espécime amostrado, foram anotados o número de identificação, a data, o local e o horário.

Todo o material coletado encontra-se depositado no Laboratório de Biologia da Universidade da Região de Joinville, em Joinville, Santa Catarina.

Os dados do levantamento de espécies de abelhas e plantas foram organizados em uma tabela que inclui, para cada táxon de Apidae amostrado, a espécie vegetal à qual estava associado.

Para avaliar a estrutura da comunidade, foram calculados os índices de Shannon-Wiener e Simpson (diversidade) e Pielou (equitatividade J) (Magurran 1988), cujos gráficos ensejaram a observação dos padrões que se desenvolvem na comunidade. Visando situar a composição específica encontrada no cenário de inventários já descritos, foi empreendido o cotejamento com trabalhos realizados em ambientes assemelhados ou próximos.

\section{RESULTADOS}

Foram amostrados 934 indivíduos de abelhas, pertencentes a 72 táxons e 27 gêneros de Colletinae, Andreninae, Halictinae, Megachilinae e Apinae (Tab. I). A ausência de chaves dicotômicas para alguns grupos de abelhas impediu a identificação até o nível específico e assim estas permaneceram como morfo-espécies. A riqueza da fauna de abelhas de São Bento do Sul, expressa para as famílias, apresentada de forma decrescente, foi: Apinae > Halictinae > Megachilinae > Andreninae $=$ Colletinae. Para a abundância, em relação às famílias, a ordem decrescente encontrada foi: Apinae > Halictinae $>$ Megachilinae $>$ Andreninae $>$ Colletinae.

Com relação à abundância de indivíduos, predominaram: Meliponina (40,8\%), Apina (26\%), Ceratinina (14,8\%) e Augochlorini $(9,7 \%)$. No que se refere à riqueza de espécies foram obtidos: Augochlorini (33,3\%), Meliponina (12,5\%), Ceratinina $(16,7 \%)$ e Halictini $(9,7 \%)$. Apis mellifera, espécie exótica, correspondeu a $26 \%$ de todas as abelhas amostradas e a 31\% dos Apinae.

As plantas amostradas somaram 44 táxons (Tab. I), pertencentes a 18 famílias, prevalecendo Asteraceae (50\% dos táxons), seguida de Begoniaceae, Iridaceae, Malvaceae, Melastomataceae e Oxalidaceae (4,5\% cada uma). Em Asteraceae, os táxons mais atrativos foram Bidens pilosa Linneus (23 espécies de abelhas), seguido por Bidens sulphurea (Cav.) Sch. Bip. e Vernoanthura tweediana Baker (14 táxons de abelhas cada). Quatro espécies estiveram floridas por maior período: Impatiens waleriana Hook (Balsaminaceae) e em Asteraceae, Bidens pilosa, Crepis japonica (Linneus) Benth e Sonchus asper (Linneus) Hill. Houve variação no número de táxons de plantas visitadas ao longo do ano (primavera: 18, verão: 31, outono: 22 e inverno: 22) e a flutuação dos valores de riqueza de táxons de abelhas e de plantas esteve correlacionada ao longo do ano (Fig. 1). 


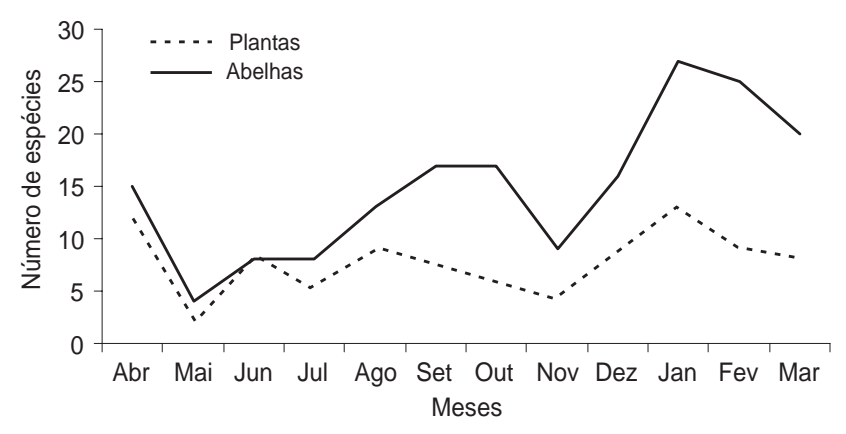

Figura 1. Frequência absoluta de espécies de abelhas e de plantas ao longo dos meses em São Bento do Sul.
Poucas espécies de plantas foram visitadas por muitas espécies de abelhas e muitas espécies de plantas foram visitadas por poucas espécies de abelhas (Fig. 2). Na comunidade de abelhas, $47 \%$ dos táxons estiveram representados por apenas um indivíduo (singletons), correspondendo a menos de $4 \%$ dos indivíduos e os táxons dominantes perfizeram 15\% do total.

Para um total de 72 horas de esforço de coleta, foram obitidos 72 táxons de abelhas e 934 indivíduos, ou seja, um esforço de captura de um táxon de abelha por hora ou 12,97 indivíduos/hora. A construção da curva de frequências acumuladas (curva do coletor) se mostrou ainda crescente.

Os valores calculados para os índices de Simpson, Shannon-Wiener e Pielou estão dispostos na tabela II e nas figuras 3-4.

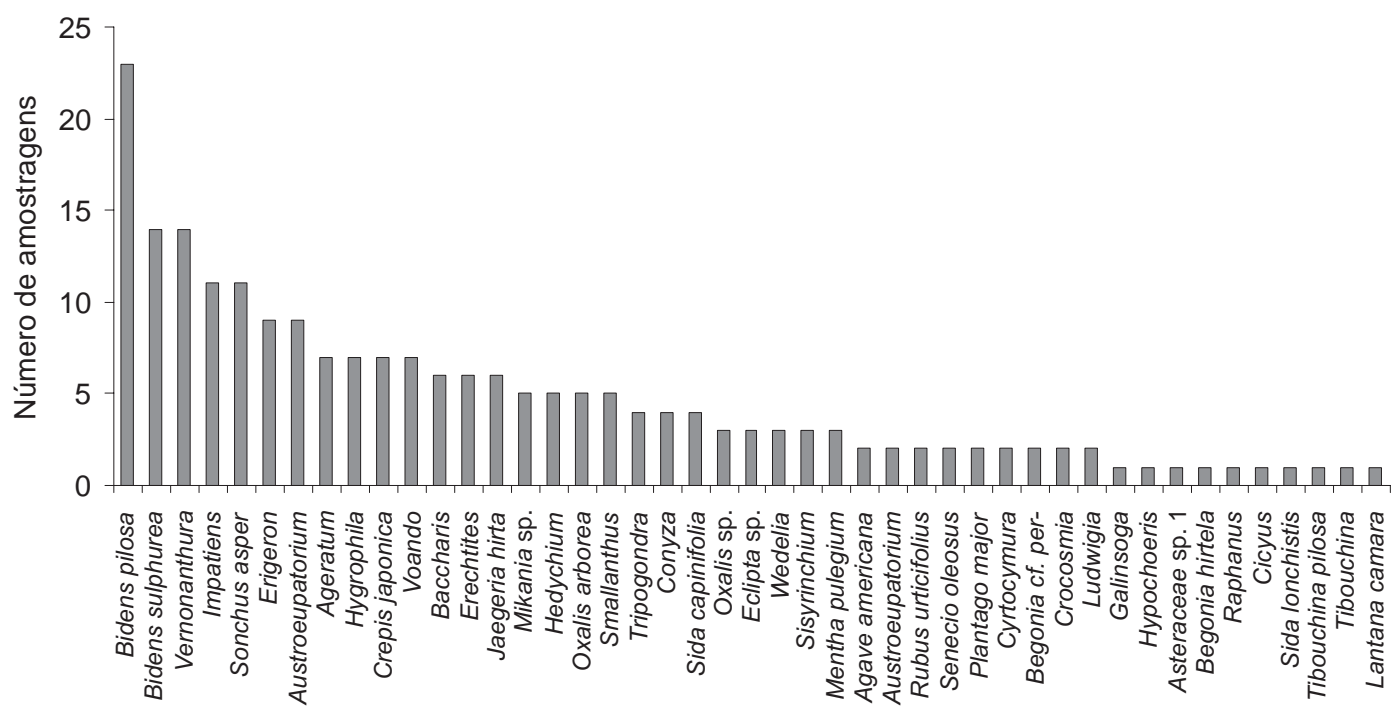

Espécies de plantas

Figura 2. Espécies de plantas e frequência de visitas por abelhas em São Bento do Sul. Espécimes em vôo foram incluídos sob a denominação voando.
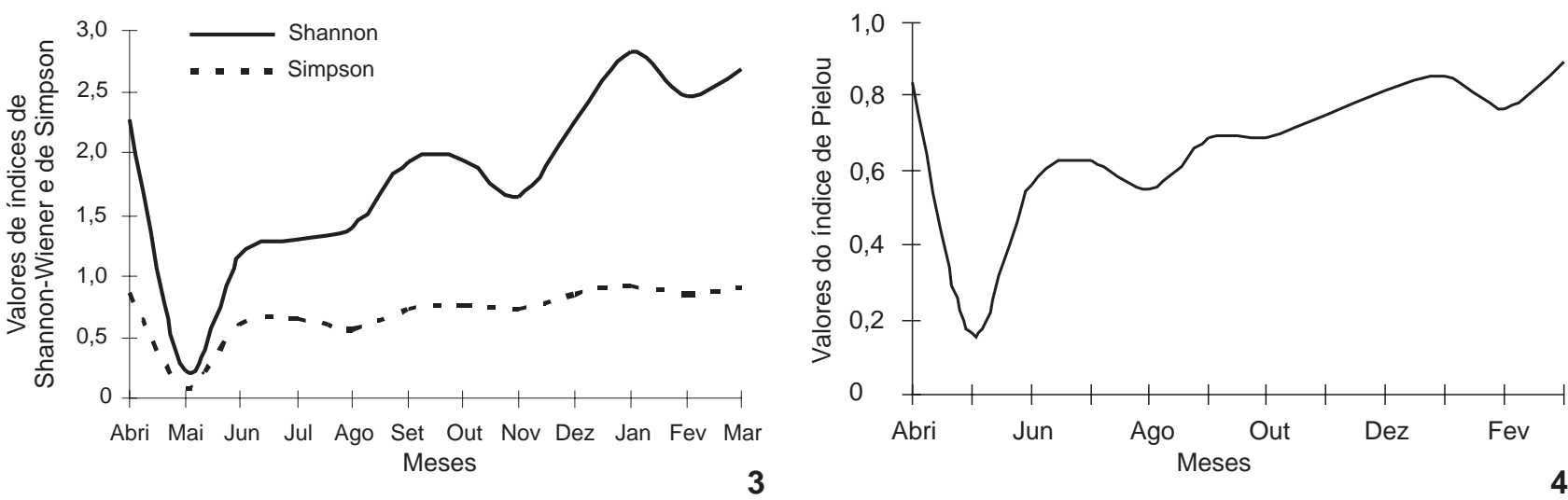

Figuras 3-4. Variação média mensal dos índices de diversidade de Shannon-Wiener e Simpson (3) e do índice de equitabilidade de Pielou (4), durante o período de 2006 a 2007, em São Bento do Sul. 
Tabela I. Lista dos táxons de abelhas, táxons de plantas associados, abundância de abelhas coletadas (N) e meses de amostragem em São

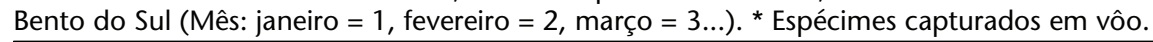

\begin{tabular}{|c|c|c|c|c|c|}
\hline \multicolumn{2}{|r|}{ Abelhas } & \multicolumn{2}{|r|}{ Plantas } & \multirow{2}{*}{$\frac{N}{1}$} & \multirow{2}{*}{$\begin{array}{c}\text { Mês } \\
12\end{array}$} \\
\hline Colletinae & cf. Hylaeus sp. & Begoniaceae & Begonia cf. per-dusenii Brade & & \\
\hline & Lonchopria cingulata Moure, 1956 & Asteraceae & Mikania sp. & 2 & 8 \\
\hline \multirow[t]{4}{*}{ Andreninae } & Anthrenoides meridionalis (Schrottky, 1902) & Oxalidaceae & Oxalis arborea R.Br. & 1 & 9 \\
\hline & & Voando* & & 1 & 10 \\
\hline & Anthrenoides politus Urban, 2005 & Asteraceae & Erechtites valerianifolius (Link ex Spreng.) DC & 4 & 12 \\
\hline & & Iridaceae & Sisyrinchium palmifolium L. & 1 & 12 \\
\hline \multirow[t]{33}{*}{ Halictinae } & Augochlora aff. cydippe (Schrottky, 1910) & Asteraceae & Bidens pilosa $\mathrm{L}$. & 1 & 1 \\
\hline & & & Eclipta sp. & 1 & 1 \\
\hline & Augochlora dolichocephala (Moure, 1941) & Asteraceae & Bidens pilosa $\mathrm{L}$. & 3 & 2 \\
\hline & Augochlora foxiana Cockerell, 1900 & Onagraceae & Ludwigia tomentosa (Camb.) Hara. & 1 & 4 \\
\hline & Augochlora semiramis (Schrottky, 1910) & Asteraceae & Austroeupatorium picturatum (Malme) King \& Rob. & 1 & 12 \\
\hline & & & Bidens pilosa $\mathrm{L}$. & 2 & 1,6 \\
\hline & & & Bidens sulphurea (Cav.) Sch. Bip & 1 & 1 \\
\hline & & & Crepis japonica (L.) Benth. & 6 & 12 \\
\hline & & & Erechtites valerianifolius (Link ex Spreng.) DC. & 1 & 8 \\
\hline & & & Vernonanthura tweediana Baker & 2 & 9,1 \\
\hline & Augochlora sp. 1 & Asteraceae & Crepis japonica (L.) Benth. & 1 & 7 \\
\hline & Augochlora sp. 2 & Asteraceae & Austroeupatorium picturatum (Malme) King \& Rob. & 1 & 12 \\
\hline & Augochlora sp. 3 & Asteraceae & Smallanthus connatus (Spreng.) H. Rob. & 1 & 1 \\
\hline & & & Bidens pilosa $\mathrm{L}$. & 1 & 2 \\
\hline & Augochlora sp. 5 & Asteraceae & Bidens pilosa $\mathrm{L}$. & 4 & $6,2,3$ \\
\hline & & & Bidens sulphurea (Cav.) Sch. Bip & 2 & 1 \\
\hline & & & Erechtites valerianifolius (Link ex Spreng.) DC. & 1 & 12 \\
\hline & & & Smallanthus connatus (Spreng.) H. Rob. & 1 & 1 \\
\hline & & & Vernonanthura tweediana Baker & 6 & 9,1 \\
\hline & & Balsaminaceae & Impatiens walleriana Hook. & 3 & 10,12 \\
\hline & & Lamiaceae & Mentha pulegium L. & 1 & 8 \\
\hline & Augochlora sp. 6 & Asteraceae & Ageratum conyzoides L. & 2 & 3 \\
\hline & & & Erigeron maximus (D. Don) DC. & 2 & 1 \\
\hline & & & Bidens pilosa $\mathrm{L}$. & 5 & 2,3 \\
\hline & Augochlora sp. 7 & Asteraceae & Ageratum conyzoides L. & 1 & 3 \\
\hline & & & Bidens sulphurea (Cav.) Sch. Bip & 1 & 1 \\
\hline & & & Bidens pilosa $\mathrm{L}$. & 7 & 2,3 \\
\hline & & & Sonchus asper (L.) Hill & 1 & 2 \\
\hline & Augochlorella ephyra (Schrottky, 1910) & Asteraceae & Bidens pilosa $\mathrm{L}$. & 1 & 10 \\
\hline & & & Erechtites valerianifolius (Link ex Spreng.) DC. & 1 & 12 \\
\hline & Augochloropsis cognata Moure, 1944 & Asteraceae & Conyza canadensis (L.) Cronquist (L.) Cronquist & 1 & 12 \\
\hline & & & Sonchus asper (L.) Hill & 1 & 2 \\
\hline & & Begoniaceae & Begonia cf. per-dusenii Bradei & 1 & 12 \\
\hline
\end{tabular}


Tabela I. Continuação.

\begin{tabular}{|c|c|c|c|c|c|}
\hline \multicolumn{2}{|r|}{ Abelhas } & \multicolumn{2}{|r|}{ Plantas } & \multirow{2}{*}{$\begin{array}{c}N \\
1\end{array}$} & \multirow{2}{*}{ Mês } \\
\hline & Augochloropsis paranaense [in litt.] & Asteraceae & Bidens pilosa $\mathrm{L}$. & & \\
\hline & Augochloropsis sp. 1 & Asteraceae & Ageratum conyzoides L. & 1 & 3 \\
\hline \multirow{2}{*}{\multicolumn{2}{|c|}{ Augochloropsis sp. 2}} & \multirow[t]{2}{*}{ Asteraceae } & Bidens pilosa $\mathrm{L}$. & 1 & 10 \\
\hline & & & Jaegeria hirta (Lag.) Less. & 1 & 4 \\
\hline & Augochloropsis sp. 3 & Asteraceae & Baccharis brachylaenoides DC. & 1 & 4 \\
\hline & Augochloropsis sp. 5 & Asteraceae & Jaegeria hirta (Lag.) Less. & 1 & 4 \\
\hline & Augochloropsis sp. 6 & Commelinaceae & Tripogandra diuretica (Mart.) Handlos & 1 & 2 \\
\hline & Ariphanarthra palpalis Moure, 1951 & Zingiberaceae & Hedychium coronarium J.König & 1 & 5 \\
\hline & Neocorynura atromarginata (Cockerell, 1901) & Acanthaceae & Hygrophila brasiliensis (Spreng.) Lindau & 1 & 2 \\
\hline & Neocorynura chapadicola (Cockerell, 1901) & Acanthaceae & Hygrophila brasiliensis (Spreng.) Lindau & 1 & 2 \\
\hline & \multirow[t]{7}{*}{ Neocorynura oiospermi (Schrottky, 1909) } & \multirow[t]{6}{*}{ Asteraceae } & Bidens pilosa $\mathrm{L}$. & 8 & $1,12,2$ \\
\hline & & & Bidens sulphurea (Cav.) Sch. Bip & 1 & 1 \\
\hline & & & Eclipta sp. & 1 & 1 \\
\hline & & & Jaegeria hirta (Lag.) Less. & 1 & 4 \\
\hline & & & Smallanthus connatus (Spreng.) H. Rob. & 1 & 1 \\
\hline & & & Wedelia paludosa DC. & 1 & 2 \\
\hline & & Malvaceae & Sida capinifolia $\mathrm{L}$. & 1 & 4 \\
\hline & Dialictus sp.1 & Asteraceae & Conyza canadensis (L.) Cronquist & 1 & 12 \\
\hline & Dialictus sp.2 & Asteraceae & Erigeron maximus (D. Don) DC. & 1 & 1 \\
\hline & Dialictus sp. 3 & Asteraceae & Bidens pilosa $\mathrm{L}$. & 1 & 1 \\
\hline & Dialictus sp.4 & Asteraceae & $\begin{array}{l}\text { Austroeupatorium picturatum (Malme) } \\
\text { King \& Rob. }\end{array}$ & 1 & 12 \\
\hline & \multirow[t]{2}{*}{ Dialictus sp.5 } & \multirow[t]{2}{*}{ Asteraceae } & $\begin{array}{l}\text { Erechtites valerianifolius (Link ex Spreng.) } \\
\text { DC. }\end{array}$ & 1 & 12 \\
\hline & & & Erigeron maximus (D. Don) DC. & 1 & 1 \\
\hline & Dialictus sp. 6 & Asteraceae & Galinsoga parviflora Cav. & 2 & 1 \\
\hline & Dialictus sp.7 & Asteraceae & $\begin{array}{l}\text { Erechtites valerianifolius (Link ex Spreng.) } \\
\text { DC. }\end{array}$ & 1 & 12 \\
\hline & Rhectomia mourei (Eickwort, 1969) & Oxalidaceae & Oxalis sp. & 1 & 9 \\
\hline & Temnosoma sp. & Iridaceae & Sisyrinchium palmifolium L. & 1 & 1 \\
\hline \multirow[t]{8}{*}{ Megachilinae } & \multirow[t]{3}{*}{$\begin{array}{l}\text { Megachile (Trichurochile) thygaterella Schrottky, } \\
1913\end{array}$} & \multirow[t]{2}{*}{ Asteraceae } & Eclipta sp. & 1 & 1 \\
\hline & & & Vernonanthura tweediana Baker & 1 & 9 \\
\hline & & Plantaginaceae & Plantago major L. & 1 & 9 \\
\hline & \multirow[t]{4}{*}{$\begin{array}{l}\text { Megachile (Moureapis) anthidioides Radoszkowski, } \\
1874\end{array}$} & \multirow[t]{3}{*}{ Asteraceae } & $\begin{array}{l}\text { Austroeupatorium picturatum (Malme) } \\
\text { King \& Rob. }\end{array}$ & 1 & 12 \\
\hline & & & Cyrtocymura scorpioides (Lam.) H.Rob. & 1 & 12 \\
\hline & & & Vernonanthura tweediana Baker & 3 & 9 \\
\hline & & Balsaminaceae & Impatiens walleriana Hook. & 2 & 10 \\
\hline & Megachile (Ptilosarus) cf. bertonii Schrottky, 1908 & Asteraceae & Mikania sp. & 1 & 3 \\
\hline
\end{tabular}


Tabela I. Continuação.

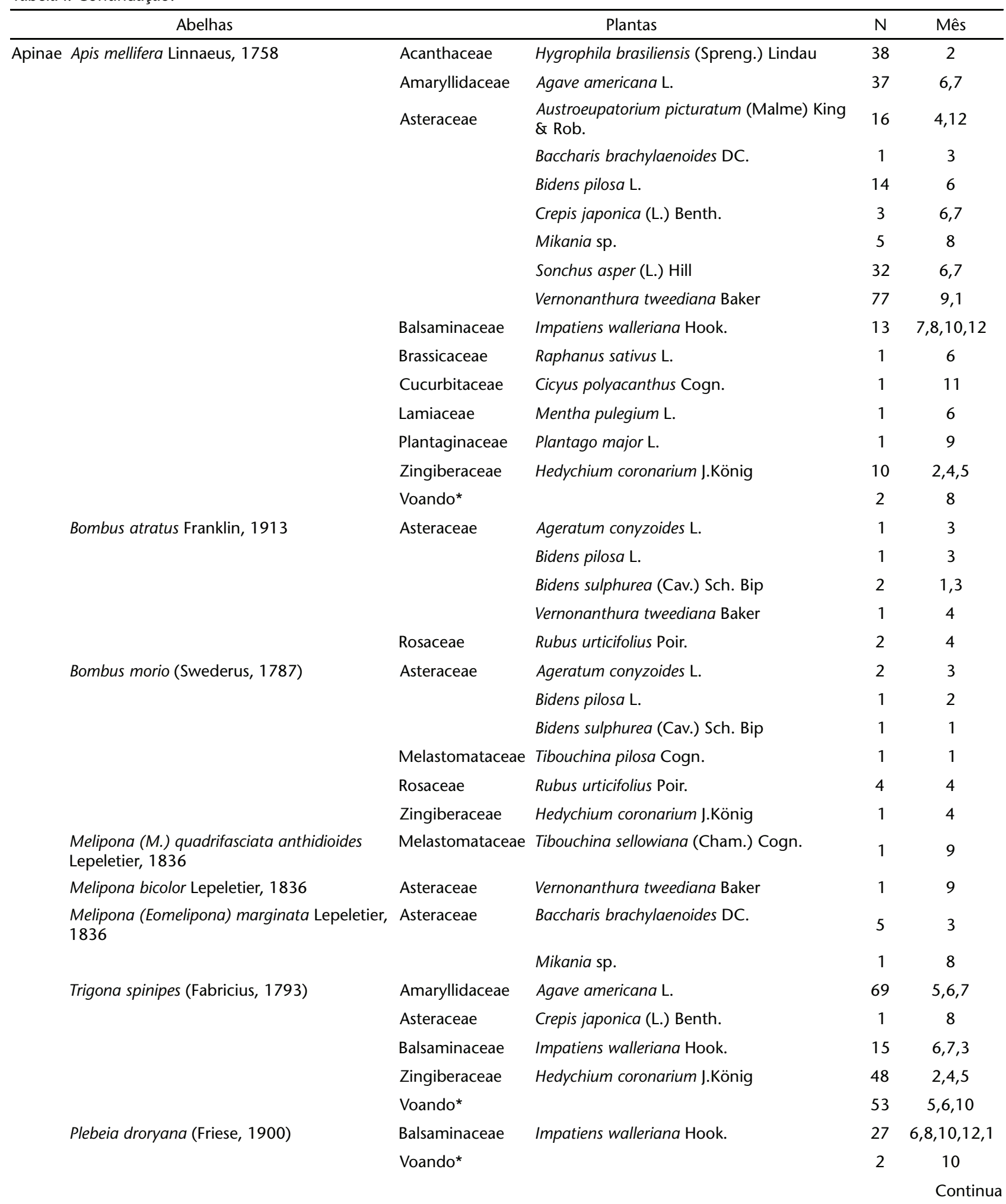


Tabela I. Continuação.

\begin{tabular}{|c|c|c|c|c|}
\hline Abelhas & & Plantas & $\mathrm{N}$ & Mês \\
\hline \multirow[t]{12}{*}{ Plebeia saiqui (Friese, 1900) } & Acanthaceae & Hygrophila brasiliensis (Spreng.) Lindau & 2 & 2 \\
\hline & \multirow[t]{8}{*}{ Asteraceae } & $\begin{array}{l}\text { Austroeupatorium picturatum (Malme) King } \\
\text { \& Rob. }\end{array}$ & 1 & 12 \\
\hline & & Baccharis brachylaenoides DC. & 1 & 3 \\
\hline & & Bidens pilosa $\mathrm{L}$. & 1 & 2 \\
\hline & & Bidens sulphurea (Cav.) Sch. Bip & 1 & 1 \\
\hline & & Crepis japonica (L.) Benth. & 7 & 7,9 \\
\hline & & Erigeron maximus (D. Don) DC. & 2 & 1 \\
\hline & & Sonchus asper (L.) Hill & 10 & $6,7,2$ \\
\hline & & Vernonanthura tweediana Baker & 2 & 9 \\
\hline & Balsaminaceae & Impatiens walleriana Hook. & 109 & $7,8,10,12,1,3$ \\
\hline & Iridaceae & $\begin{array}{l}\text { Crocosmia crocosmiiflora (W. A. Nicholson) } \\
\text { N.E.Br. }\end{array}$ & 1 & 11 \\
\hline & Voando* & & 6 & $5,7,10$ \\
\hline Tetragonisca angustula (Latreille, 1811) & Asteraceae & Sonchus asper (L.) Hill & 1 & 2 \\
\hline \multirow[t]{4}{*}{ Scaptotrigona bipunctata (Lepeletier, 1836) } & Acanthaceae & Hygrophila brasiliensis (Spreng.) Lindau & 8 & 2 \\
\hline & \multirow[t]{3}{*}{ Asteraceae } & Ageratum conyzoides L. & 1 & 3 \\
\hline & & Baccharis brachylaenoides DC. & 2 & 3 \\
\hline & & Wedelia paludosa DC. & 1 & 2 \\
\hline Oxytrigona sp. & Asteraceae & Baccharis brachylaenoides DC. & 1 & 3 \\
\hline \multirow[t]{2}{*}{ Ceratina (Calloceratina) sp. 1} & Asteraceae & Vernonanthura tweediana Baker & 1 & 10 \\
\hline & Verbenaceae & Lantana camara L. & 1 & 8 \\
\hline Ceratina (Calloceratina) sp. 2 & Iridaceae & Sisyrinchium palmifolium L. & 1 & 1 \\
\hline Ceratina (Calloceratina) sp. 3 & Asteraceae & Vernonanthura tweediana Baker & 1 & 10 \\
\hline \multirow[t]{2}{*}{ Ceratina (Ceratinula) sp. 1} & Asteraceae & Sonchus asper (L.) Hill & 1 & 9 \\
\hline & Oxalidaceae & Oxalis sp. & 2 & 9,1 \\
\hline \multirow[t]{4}{*}{ Ceratina (Crewella) sp. 2} & Asteraceae & Bidens pilosa $\mathrm{L}$. & 1 & 3 \\
\hline & & Bidens sulphurea (Cav.) Sch. Bip & 1 & 1 \\
\hline & & Senecio oleosus Klatt. & 2 & 4 \\
\hline & Onagraceae & Ludwigia tomentosa (Camb.) Hara. & 1 & 4 \\
\hline \multirow[t]{11}{*}{ Ceratina (Crewella) sp. 3} & Asteraceae & Austroeupatorium inulaefolium Kunth & 1 & 3 \\
\hline & & $\begin{array}{l}\text { Austroeupatorium picturatum (Malme) King } \\
\text { \& Rob. }\end{array}$ & 1 & 12 \\
\hline & & Bidens pilosa $\mathrm{L}$. & 3 & $1,10,12$ \\
\hline & & Bidens sulphurea (Cav.) Sch. Bip & 4 & 1 \\
\hline & & Conyza canadensis (L.) Cronquist & 1 & 12 \\
\hline & & Erigeron maximus (D. Don) DC. & 6 & 1 \\
\hline & & Hypochoeris brasiliensis (Less.) Benth. & 1 & 12 \\
\hline & & Jaegeria hirta (Lag.) Less. & 1 & 2 \\
\hline & & Sonchus asper (L.) Hill & 2 & 6,9 \\
\hline & & Vernonanthura tweediana Baker & 9 & 9,1 \\
\hline & & & & Continua \\
\hline
\end{tabular}


Tabela I. Continuação.

\begin{tabular}{|c|c|c|c|c|}
\hline Abelhas & & Plantas & $\mathrm{N}$ & Mês \\
\hline & & Wedelia paludosa DC. & 1 & 2 \\
\hline & Balsaminaceae & Impatiens walleriana Hook. & 1 & 1 \\
\hline & Lamiaceae & Mentha pulegium L. & 1 & 7 \\
\hline & Oxalidaceae & Oxalis arborea R.Br. & 2 & 9 \\
\hline & Voando* & & 1 & 7 \\
\hline \multirow[t]{4}{*}{ Ceratina (Crewella) sp. 3} & Asteraceae & Bidens pilosa $\mathrm{L}$. & 2 & 2,3 \\
\hline & & Bidens sulphurea (Cav.) Sch. Bip & 2 & 1 \\
\hline & & Sonchus asper (L.) Hill & 1 & 8 \\
\hline & Commelinaceae & Tripogandra diuretica (Mart.) Handlos & 1 & 2 \\
\hline Ceratina (Crewella) sp. 6 & Balsaminaceae & Impatiens walleriana Hook. & 1 & 7 \\
\hline Ceratina (Crewella) sp. 8 & Asteraceae & Erigeron maximus (D. Don) DC. & 1 & 1 \\
\hline \multirow[t]{14}{*}{ Ceratina (Crewella) sp. 9} & Acanthaceae & Hygrophila brasiliensis (Spreng.) Lindau & 2 & 2 \\
\hline & Asteraceae & Ageratum conyzoides L. & 1 & 3 \\
\hline & & Bidens pilosa $\mathrm{L}$. & 15 & $, 2,3,6,8,10$ \\
\hline & & Bidens sulphurea (Cav.) Sch. Bip & 7 & 1,3 \\
\hline & & Crepis japonica (L.) Benth. & 2 & 8,9 \\
\hline & & Conyza canadensis (L.) Cronquist & 1 & 12 \\
\hline & & Cyrtocymura scorpioides (Lam.) H.Rob. & 1 & 12 \\
\hline & & Erigeron maximus (D. Don) DC. & 2 & 1 \\
\hline & & Jaegeria hirta (Lag.) Less. & 3 & 2 \\
\hline & & Sonchus asper (L.) Hill & 1 & 6 \\
\hline & & Vernonanthura tweediana Baker & 4 & $2,9,10$ \\
\hline & Iridaceae & Crocosmia crocosmiiflora (W.A. Nicholson) N.E.Br. & 1 & 9 \\
\hline & Oxalidaceae & Oxalis arborea R.Br. & 3 & $9,10,12$ \\
\hline & Voando* & & 1 & 6 \\
\hline \multirow[t]{15}{*}{ Ceratina (Crewella) sp. 9} & Asteraceae & Austroeupatorium inulaefolium Kunth & 4 & 3 \\
\hline & & Bidens pilosa $\mathrm{L}$. & 13 & $1,2,3$ \\
\hline & & Bidens sulphurea (Cav.) Sch. Bip & 3 & 1,3 \\
\hline & & Crepis japonica (L.) Benth. & 1 & 7 \\
\hline & & Erigeron maximus (D. Don) DC. & 2 & 1,3 \\
\hline & & Hypochoeris brasiliensis (Less.) Benth. & 1 & 1 \\
\hline & & Jaegeria hirta (Lag.) Less. & 1 & 2 \\
\hline & & Sonchus asper (L.) Hill & 1 & 8 \\
\hline & & Vernonanthura tweediana Baker & 3 & 9,1 \\
\hline & & Sp.1 & 1 & 9 \\
\hline & Balsaminaceae & Impatiens walleriana Hook. & 3 & $1,7,10$ \\
\hline & Begoniaceae & Begonia hirtela & 1 & 1 \\
\hline & Commelinaceae & Tripogandra diuretica (Mart.) Handlos & 2 & 2 \\
\hline & Malvaceae & Sida capinifolia L. & 1 & 4 \\
\hline & Oxalidaceae & Oxalis arborea R.Br. & 1 & 9 \\
\hline
\end{tabular}


Tabela I. Continuação.

\begin{tabular}{|c|c|c|c|c|}
\hline Abelhas & & Plantas & $\mathrm{N}$ & Mês \\
\hline Ceratina (Crewella) sp. 13 & Oxalidaceae & Oxalis sp. & 1 & 9 \\
\hline Xylocopa artifex Smith, 1874 & Commelinaceae & Tripogandra diuretica (Mart.) Handlos & 1 & 4 \\
\hline Xylocopa brasilianorum (Linnaeus, 1767) & Zingiberaceae & Hedychium coronarium J.König & 1 & 1 \\
\hline Xylocopa frontalis (Olivier, 1789) & Asteraceae & Smallanthus connatus (Spreng.) H. Rob. & 1 & 1 \\
\hline Thygater (Nectarodiaeta) tuberculata Urban, 1967 & Asteraceae & Vernonanthura tweediana Baker & 1 & 9 \\
\hline Melissoptila aureocincta Urban, 1968 & Asteraceae & $\begin{array}{l}\text { Austroeupatorium picturatum (Malme) } \\
\text { King \& Rob. }\end{array}$ & 1 & 12 \\
\hline \multirow[t]{6}{*}{ Melissoptila thoracica (Smith, 1854) } & Asteraceae & Bidens sulphurea (Cav.) Sch. Bip & 4 & 1 \\
\hline & & Erigeron maximus (D. Don) DC. & 2 & 1 \\
\hline & & Smallanthus connatus (Spreng.) H. Rob. & 1 & 1 \\
\hline & Malvaceae & Sida capinifolia $\mathrm{L}$. & 1 & 4 \\
\hline & & Sida lonchitis St. Hil. \& Naud. & 1 & 1 \\
\hline & Oxalidaceae & Oxalis arborea R.Br. & 1 & 10 \\
\hline \multirow[t]{3}{*}{ Paratetrapedia fervida (Smith, 1879) } & Acanthaceae & Hygrophila brasiliensis (Spreng.) Lindau & 3 & 4 \\
\hline & Asteraceae & Bidens sulphurea (Cav.) Sch. Bip & 1 & 1 \\
\hline & & Senecio oleosus Klatt. & 1 & 1 \\
\hline Paratetrapedia volatilis (Smith, 1879) & Asteraceae & Bidens pilosa $\mathrm{L}$. & 2 & 2 \\
\hline \multirow[t]{2}{*}{ Lophopedia sp. } & Balsaminaceae & Impatiens walleriana Hook. & 1 & 7 \\
\hline & Malvaceae & Sida capinifolia $\mathrm{L}$. & 1 & 4 \\
\hline Leiopodus sp. & Asteraceae & Mikania sp. & 1 & 8 \\
\hline
\end{tabular}

Tabela II. Frequência absoluta de indivíduos e de espécies de abelhas em São Bento do Sul, em 2006-2007, e índices de diversidade (Shannon-Wiener e Simpson) e equitabilidade J (Pielou).

\begin{tabular}{|c|c|c|c|c|c|c|c|c|c|c|c|c|}
\hline Parâmetros & Abri & Mai & Jun & Jul & Ago & Set & Out & Nov & Dez & Jan & Fev & Mar \\
\hline Número de indivíduos & 43 & 91 & 103 & 111 & 62 & 77 & 98 & 37 & 55 & 77 & 120 & 60 \\
\hline Número de espécies & 15 & 4 & 8 & 8 & 13 & 17 & 17 & 9 & 26 & 27 & 25 & 20 \\
\hline Shannon-Wiener & 2,266 & 0,226 & 1,169 & 1,3 & 1,407 & 1,943 & 1,955 & 1,651 & 2,26 & 2,823 & 2,469 & 2,679 \\
\hline Simpson & 0,846 & 0,085 & 0,610 & 0,665 & 0,573 & 0,720 & 0,763 & 0,729 & 0,853 & 0,916 & 0,850 & 0,912 \\
\hline Pielou & 0,837 & 0,163 & 0,562 & 0,625 & 0,548 & 0,685 & 0,690 & 0,751 & 0,815 & 0,856 & 0,767 & 0,894 \\
\hline
\end{tabular}

\section{DISCUSSÃO}

A ordem decrescente de representatividade das famílias, tanto de riqueza de espécies quanto de abundância de indivíduos, foi praticamente igual. A abundância de Apinae deveu-se principalmente à presença de $A$. mellifera, que tem sido notada na grande maioria dos censos realizados (PInHeiroMACHADO et al. 2006). Comparando os resultados encontrados neste trabalho com inventários faunísticos variados do sul do Brasil (BARbola \& LaRoca 1993, ORTOLAN \& LaRoca 1996) verificase que a distribuição do número de espécies entre Apidae segue praticamente o mesmo padrão. Apinae e Halictinae são as subfamílias com maior riqueza de espécies e de indivíduos em diversos trabalhos, sendo que Halictinae provavelmente tem sua origem biogeográfica nesta área do continente (KRUG \& Alves-dos-Santos 2008).

Entretanto, a compreensão dos padrões de distribuição da fauna de abelhas do Brasil encontra obstáculos de síntese, tais como: a dificuldade em solucionar problemas taxonômicos existentes e, principalmente, a falta de publicações com os dados resultantes (PinheIro-MAChado et al. 2006).

Os grupos de abelhas de maior prevalência no ambiente (Meliponina, Ceratinina e Halictinae) são, o primeiro, típico de formações vegetais de mata e, os dois últimos, de expressão 
na região meridional, acompanhando os dados de Alves-DosSANTOS (2007).

Dentre as espécies de abelhas encontradas, foram observados diversos táxons não assinalados para Santa Catarina (18\%), assim como, vários designados na literatura (Moure et al. 2007, Silveira et al. 2002) apenas para os estados vizinhos: Augochlora cydipe Schrottky, 1910, A. dolichocephala (Moure, 1941), A. foxiana Cockerell, 1910, A. semiramis Schrottky, 1910, Augochloropsis cognata Moure, 1944 (Augochlorini); Megachile (T.) thygaterella Schrottky, 1913, Megachile (P.) bertonii Schrottky, 1908 (Megachilinae); Plebeia droryana (Friese, 1900), P. saiqui (Friese, 1900), Scaptotrigona bipunctata (Lepeletier, 1836) (Meliponina); Lonchopria cingulata Moure, 1956 (Colletinae) ou para estados distantes como Thygater (N.) tuberculata Urban, 1967 (Eucerini), do Rio de Janeiro. Estas duas últimas espécies reportadas como de ocorrência rara.

Espécies indicativas de ambientes bem preservados ou recentemente descritas foram também notadas: Melipona bicolor Lepeletier, 1836 (Meliponina), Anthrenoides pollitus Urban, 2005 (Andreninae); Rhectomia mourei (Eickwort, 1969) e Augochloropsis paranaensis [in litt.] (Augochlorini).

A nítida preferência floral por Asteraceae, família botânica mais representativa nas análises, representa uma predominância de escolha floral recorrente na parte meridional do país (Pinheiro-Machado et al. 2006).

A observação tanto de espécies ruderais quanto típicas de FODM de altitude corrobora as informações de outros autores (Steiner et al. 2006) da importância do primeiro tipo de vegetação como pasto apícola para as abelhas.

O padrão de flutuação dos valores de riqueza de espécies de abelhas e de plantas confirmou o encontrado por outros autores (SILvEIRA et al. 2002), de que há uma correlação entre os dois fatores. Por outro lado, a maior presença de abelhas sobre as flores nas estações mais quentes indica a sazonalidade da comunidade, padrão esperado para a latitude.

O padrão de exploração dos recursos florais encontrado neste trabalho é reportado como frequente em estudos de comunidades de abelhas (Pinheiro-Machado et al. 2006). Os dados encontrados sugerem que ocorrem possivelmente relações de oligolectia. Alguns poucos recursos florais mostraram ser unanimidade entre as espécies de abelhas e outros medianamente explorados (RoubIK 1989).

A estrutura de comunidade avaliada sugere uma guilda com forte preponderância de alguns táxons, que se evidencia pela presença de singletons e espécies dominantes. O valor encontrado de esforço de captura apresentou-se relativamente elevado.

Na análise da variação dos índices, o de Simpson, que atribui maior peso a espécies raras, evidenciou uma dominância relativamente baixa entre os táxons (padrão de partição de recursos relativamente igualitário) e o de Shannon-Wiener, que pondera espécies raras e comuns, mostrou oscilação na diversidade, sugerindo influência sazonal, com aumento em direção aos períodos de maiores temperaturas, seguido de uma diminuição nos meses de inverno. O índice de Pielou revelou um padrão crescente, de relativo equilíbrio, com exceção de um momento. Trabalhos realizados para verificar a composição de comunidades de abelhas têm demonstrado padrões ora assemelhados ora distintos, sugerindo a necessidade de estudos de maior duração temporal (PINHEIro-MACHADo et al. 2006). Há que se fazer a ressalva da influência do padrão sazonal e da espécie introduzida $A$. mellifera, amplamente preponderante em alguns momentos, o que já foi notado por outros autores (STEINER et al. 2006).

O pequeno número de espécies abundantes e a alta proporção de espécies representadas por poucos indivíduos, assim como, a variação destes parâmetros em relação às estações, indicam um padrão de distribuição do tipo transicional entre regiões subtropical e temperada. As faixas de frequências reveladas pelos índices indicam um local com diversidade relativamente alta, não completamente registrada neste trabalho.

A escolha floral da apifauna estudada neste trabalho, acompanhou o padrão fenológico das plantas da região. SAKAGAMI et al. (1967), na publicação que consagraria a metodologia dos levantamentos de abelhas, observaram que no sul do Brasil, a variação sazonal do número de espécies vegetais visitadas reflete as flutuações de temperatura.

As abelhas são polinizadoras de ambientes naturais e cultivados. Sua distribuição temporal reflete os padrões estacionais e a intensidade de polinização acompanha este movimento. Por outro lado, os problemas decorrentes do uso mono-específico de polinizadores em agricultura tem recomendado a utilização de espécies nativas de abelhas (MMA 2002). Em zona subtropical/temperada, esta recomendação deve levar em conta as implicações sazonais no caso de culturas de inverno que necessitem de polinizadores. Essa consideração denota a importância dos levantamentos de apifauna anuais que identifiquem as espécies de abelhas que mantêm atividade sem restrições ao longo das estações e que poderiam servir como alternativa à A. melifera, amplamente disseminada (KEVAN \& Phillips 2001). Esse táxon, originário de áreas temperadas, desenvolve atividade externa mesmo no inverno, produzindo grandes estoques de alimento e de prole, chegando às estações amenas com altos padrões populacionais, muito competitivos.

Esse estudo evidenciou espécies de ocorrência inédita, rara ou díspar, o que indica um local bastante intocado e que necessita de mais estudos, principalmente em vista do acompanhamento do manejo da área.

Áreas naturais protegidas servem como abrigo para espécies ameaçadas, desconhecidas, assim como, celeiro de dispersão-refúgio ligado a ambientes vizinhos inóspitos. Desta forma, a interação que existe entre as abelhas e seus recursos florais, a manutenção dos ecossistemas que dela decorre em função da polinização e a taxa de melissodiversidade verificada, confirmam seu papel como bioindicadores e recomendam práticas de preservação dos recursos naturais da área em apreço, como ferramenta integradora de conservação ambiental. 


\section{AGRADECIMENTOS}

À Vanessa A. Valentini, Hiuta R. Christovão e Iuri S.A. Anni, pelo auxílio nos trabalhos de campo. À Pró-Reitoria de Pesquisa e Pós-Graduação da UNIVILLE, pelas bolsas concedidas ao projeto desenvolvido. A Danúncia Urban, Gabriel A.R. Melo e Rodrigo Gonçalves, pelo auxílio na identificação taxonômica das abelhas. Aos pesquisadores do Herbário Joinvillea da UNIVILLE pelo auxílio na identificação botânica. A todos que contribuíram para a realização deste trabalho.

\section{LITERATURA CITADA}

Alves-dos-Santos, I. 2007. Estudos sobre comunidades de abelhas no sul do Brasil e proposta para avaliação rápida da apifauna subtropical. Brazilian Journal of Ecology 11 (12): 53-65.

Barbola, I.F. \& S. Laroca. 1993. A comunidade de Apoidea (Hymenoptera) da Reserva Passa Dois (Lapa, Paraná, Brasil): I. Diversidade, abundância relativa e atividade sazonal. Acta Biológica Paranaense 22: 91-113.

Kevan, P.G. 1999. Pollinators as bioindicators of the state of the environment: species, activity and diversity. Agriculture, Ecosystems \& Environment 74: 373-393.

Kevan, P.G. \& T.P. Phillips. 2001. The economic impact of pollinator decline: an approach to assessing the consequences. Conservation ecology. Disponível online em: http:// www.conseecol.org/vol5iss1/art8. [Acesso: 28/XII/2008].

Krug, C. \& I. Alves-dos-Santos. 2008. O Uso de Diferentes Métodos para Amostragem da Fauna de Abelhas (Hymenoptera, Apoidea), um Estudo em Floresta Ombrófila Mista em Santa Catarina. Neotropical Entomology 37 (3) 265-278.

LEwINsohn, T.M. 2001. Esboço de uma estratégia abrangente de inventários de biodiversidade, p. 376-384 In: I. GARAY; B. DIAS (Eds). Conservação da biodiversidade em ecossistemas tropicais. Petrópolis, Editora Vozes, 430p.

MAGURRAN, A.E. 1988. Ecological diversity and its measurement. Princeton, Princeton University Press, X+179p.

Martins, C.F. 1994. Comunidade de abelhas (Hymenoptera, Apoidea) da caatinga e do cerrado com elementos de campo rupestre do estado da Bahia, Brasil. Revista Nordestina de Biologia 9 (2): 225-257.

Medeiros, J.D. 2002. Mata Atlântica em Santa Catarina, p. 103109. In: Schäfer, W.B. \& M. Prochnow. A Mata Atlântica e você: como preservar, recuperar e se beneficiar da mais ameaçada floresta brasileira. Brasília, APREMAVI, 156p.

Melo, G.A.R \& R. GonçaLves. 2005. Higher-level bee classifications (Hymenoptera, Apoidea, Apidae sensu lato). Revista Brasileira de Zoologia 22 (1): 153-159.

Michener, C.D. 2000. The bees of the world. Baltimore, John Hopkins University Press, XIV+913p.

Michener, C.D.; R.J. Mcginley \& B.N. Danforth. 1994. The bee genera of north and central America (Hymenoptera: Apoidea). Washington, D.C., Smithsonian Institution Press, VIII+209p.

Mma-Ministerio do Meio Ambiente. 2002. The São Paulo Declaration on Pollinators. Disponível online em: www.mma.gov. br/biodiversidade/doc/pollinas.pdf [Acesso: 28/XII/2008].

Moure, J.S.; D. Urban \& G.A.R. Melo. 2007. Catalogue of bees (Hymenoptera, Apoidea) in the neotropical region. Curitiba, Sociedade Brasileira de Entomologia, XIV+1058p.

Ortolan, S.M.L.S. \& S. Laroca. 1996. Melissocenótica em áreas de cultivo de macieira (Pyrus malus L.) em Lages (Santa Catarina), com notas comparativas e experimento de polinização com Plebeia emerina (Friese) (Hymenoptera, Apoidea). Acta Biológica Paranaense 25: 1-113.

Pinheiro-Machado, C.; I. Alves-Dos-Santos; V.L. Imperatriz-FonseCA; A.M.P. Kleinert \& F.A. Silveira. 2006. Brazilian bee surveys: state of knowledge, conservation and sustainable use. In: P.G. Kevan \& V.L. Imperatriz-Fonseca (Eds). Pollinating bees: the conservation link between agriculture and nature. Brasília, Ministério do Meio Ambiente, $2^{\mathrm{a}}$ ed., 336p.

RoubIK, D.W. 1989. Ecology and natural history of tropical bees. New York, Cambridge University Press, X+514p.

SaKagami, S.F.; S. Laroca \& J.S. Moure. 1967. Wild bees biocenotics in São José dos Pinhais (PR), south Brazil. Preliminary report. Journal of the Faculty of Science of the Hokkaido University, Serie Zoology, 16 (2): 253-291.

Silveira, F.A.; G.A.R. Melo \& E.D.A. AlmeidA. 2002. Abelhas brasileiras. Sistemática e identificação. Belo Horizonte, Fernando A. Silveira, 253p.

Steiner, J.; B. Harter-Marques; A. Zillikens \& E.P. Feja. 2006. Bees of Santa Catarina Island, Brazil - a first survey and checklist (Insect: Apoidea). Zootaxa 1220: 1-18.

Tonhasca JR, A. 2005. Ecologia e história natural da Mata Atlântica. Rio de Janeiro, Interciência, X+197p.

Submitted: 18.XII.2008; Accepted: 21.I.2010.

Editorial responsibility: Kleber del Claro 